\title{
Multi-Channel Framework for Body Area Network in Health Monitoring
}

\author{
Nabil Ali Alrajeh ${ }^{1, *}$, Shafiullah Khan ${ }^{2}$, Carlene E-A Campbell ${ }^{3}$ and Bilal Shams ${ }^{2}$ \\ ${ }^{1}$ Biomedical Technology Department, College of Applied Medical Sciences, King Saud University, Saudi Arabia \\ ${ }^{2}$ Institute of Information Technology, Kohat University of Science and Technology (KUST), Pakistan \\ ${ }^{3}$ Swansea Metropolitan University, United Kingdom
}

Received: 3 Jan. 2013, Revised: 5 May. 2013, Accepted: 7 May. 2013

Published online: 1 Sep. 2013

\begin{abstract}
Increasing use of wireless networks has empowered the facility of ubiquitous health monitoring especially in advanced countries. Various small devices are attached to human body forming personalized wireless body area network (WBAN). These small devices interact with each other using different radios and antennas schemes proposed by many researchers around the globe. However, most of these schemes are facing problem of inefficiency due to fading and shadowing effects. We propose a novel multi-radio multichannel framework for efficient communication among devices in WBAN. The focus of this research is to ensure energy efficient and reliable communication in WBAN.
\end{abstract}

Keywords: Wireless body area network, multi-radio multi-channel, health monitoring, e-health

\section{Introduction}

A wireless sensor network (WSN) has many applications in which wireless body area network (WBAN) has gained significant importance. In WBAN [1,2], small electronic devices are attached with human to monitor specific health related problem such as blood pressure, blood sugar level, organ movement etc. The concept of WBAN is presented to facilitate the health care issues distantly. WBAN consists of small intelligent electronic devices termed as sensors, which are low in power and processing [3]. These small sensors collects heath related data and communicate that data to some medical officers or medical servers so that it can be analyzed and monitor the patient health parameters. This kind of mechanism created ease in patient life to enjoy mobility and need not to stay at hospital all the time. In WBAN, three layers play an important role for sensing precise readings of patient health and transmitting accurate information to medical servers, i.e. physical layer, MAC layer and network layer. Physical layer is concerned with energy, antennas and radios. MAC layer has the responsibility to manage data rates, which network layer has a role to search optimal route from source to destination.
Due to resource constraints, routing protocols designed for mobile ad hoc networks or wireless sensor networks cannot be used in WBAN. Research community is trying to design specialized routing protocols for WBAN such as TARA [4], ALTR [5]. These protocols are concern with body heat and to find alternate path. Few LEACH based clustered routing protocols are also presented [6]. At MAC layer, it is useful to implement wireless sensor protocols in WBAN [7].

At physical layer, WBAN normally faces significant signal loss using narrow band or ultra wide band [7]. Furthermore, less path loss is observed in line of sight (LOS) communication as compared to non line of sight due to capability of body fluid to absorb waves [8]. Fast movement of human can also greatly affect the signal loss, by increasing movement and degree of movement, more signal loss occur [9-11]. There are many research and design issues in antenna and radios that must be resolved to enable efficient and flawless deployment of WBAN. In this paper we present the possibility of multi-radio multi-channel deployment in WBAN. IEEE 802.11 free frequency band consists of many non-overlapping channels that can be linked together in multi-radio to improve the overall connectivity and

\footnotetext{
*Corresponding author e-mail: nabil@ksu.edu.sa
} 
efficiency in WBAN. This kind of mechanism is capable to increase data rates in case if multimedia information are required to analyze patient medical condition. Multi-radio multi-channel can also improve the overall end to end throughput. The rest of the paper is organized as follows. Section 2 discusses the concept of multi radio multi channel assignment in wireless sensor networks. Section 3 briefly introduces possible deployment of multi-radio multi-channel in WBAN and how it can improve the performance of WBAN. Section 4 discusses results. Finally section 5 concludes the paper.

\section{Multi-radio Multi-channel mechanism}

WBAN is currently being used in a variety of healthcare applications. In [2], WBAN architecture is proposed for physiological signal monitoring and health consulting in ubiquitous environment. In this mechanism ZigBee is used to communicate mobile system and physiological devices. Furthermore the author proposes few algorithms such as scanning, dynamic discovery and healthcare profile. Hip rehabilitation system using WBAN is proposed in [13]. In this paper, the author pointed out few challenges such as energy efficiency, reliability, network operation and low latency. Furthermore, hip rehabilitation system is proposed for such patients which suffers from hip surgery. To measure the force between hip and shoe, capacitive insole sensors are used, while magnetic sensors are used to measure hip and leg position of patients. A complete survey is conducted in [14]. The focus of this survey is to highlight few patient monitoring systems and discussion about different WBAN technologies. Furthermore, the author discusses recent and current research work done in physical, MAC and network layers of WBAN. Some security issues and mechanisms are also highlighted in this survey paper.

Ambulatory health status monitoring system with the help of software and hardware architecture is presented in [15]. This system monitors body motion and heart activities using multiple sensor nodes and personal server. In [16], WBAN challenges and opportunities are discussed in details regarding application areas, communication, storage, energy harvesting and compatibility issues. Another body area network survey is presented in [17]. In this survey, the focus of discussion is on WBAN intra-BAN and inter-BAN communication modeling, different hardware and devices, physical, MAC and network layer issues and energy conservation strategies. Furthermore, taxonomy of body sensor projects is highlighted.

There are two important components of WBAN, i.e. sensor nodes and actuators [7]. Sensor nodes sense some specific health issue while actuators are like sink nodes which take some actions based on received data from sensors. However, more end to end delay and congestion will be main concerns due to few non-overlapping channels at actuator end. If the medical condition of patient is such as which needs images or multimedia data at medical servers, in such case high data rates are needed. Most of sensor nodes operate at $2.4 \mathrm{GHz}$ frequency band and uses CSMA/CA at MAC layer. MAC layer performs some vital tasks such as data rate management, load balancing, error control, power control and collision avoidance. It should be noted that there are many contention free MAC protocols such as TDMA, FDMA etc which can perform better under centralized mechanism. However, as we know WBAN and WSN are decentralized and distributed in nature. Furthermore these contention free protocols need specialized hardware which are not suitable for WBAN or WSN.

CSMA/CA is contention based MAC protocol, it tries to reduce the collision, however it creates the issue of hidden and exposed node. Distributed Coordination Function (DCF) [18] is an enhanced protocol which is proposed to resolve those issues created by CSMA/CA. DCF uses NAV to assume future transmission based on RTC/CTS mechanism.

In multi-radio multi channel mechanisms, multiple frequency bands are used to increase the throughput and reduce end to end delay. First multi radio multi channel mechanism was proposed using graph theory [19, 20]. Multi-channel mechanism increases throughput and reduces interference among nodes.

\section{Framework}

Usually WBAN devices are equipped with very limited energy and hence the size of sensor used are very small (less than $1 \mathrm{~cm}^{3}$ ) [11]. The hardware used in proposed architecture are four small size sensors which are attached to hands and feet, and a personal server which can be smart phone or personal computer. The overall architecture is infrastructure based with star topology. The sensor nodes used are iMote2 [17] having TinyOS and follows IEEE-802.15.4 standard. The maximum range of these sensor nodes is not more than $20 \mathrm{~m}$ with a data rate of 250kbs. IEEE-801.15.4 is typical wireless personal area networks (WPAN) which operates are $2.4 \mathrm{GHz}$ frequency band. These sensor nodes are connected with personal server. The primary objective of these sensor nodes is to monitor body motion with respect to time. Body motion readings are transmitted to personal server for further processing.

In such cases, where a lot of body sensor nodes are used to cover various parts of patient body, more end to end delay and congestion would be main concerns due to few non-overlapping channels at actuator end. If the medical condition of patient is such as which needs images or multimedia data at medical servers, in such case high data 
rates are needed.

In WBAN, some factors greatly reduce the signal strength such as those sensors which are placed inside human body;most of signals are absorbed by the fluid of human body. Those sensors which are placed around human body will diverge many signals due to motion of human body or obstacles. Let suppose, one sensor node is placed at the back of patient and the other sensor is placed at the chest, as these nodes have limited signal strength, so it is difficult for a node at the back to communicate with the node at the chest. This kind of lack of communication can be minimized by using either high power nodes or to use multi-radio muti-channel mechanism in WBAN. The proposed framework is given in Figure 1. All these radios

\begin{tabular}{|c|c|c|}
\hline \multicolumn{3}{|c|}{ Media Access Control } \\
\hline Radio-A & Radio-B & Radio-C \\
Channel-A & Channel-B & Channel-C \\
\hline
\end{tabular}

Fig. 1: Proposed Framework of multi-channel for Body Area Networks

and channels are providing parameters to MAC layer. Actuator has multi radio interface which is used to receive data from each non-overlapping channel. Every sending sensor node around or inside the body has channels which are assigned to particular radio. The sensor node which is sending data to actuator uses particular channel.

\section{Results and discussion}

Successful and efficient data delivery is one of the most desirable aspects of any mechanism. Figure 2 present the data delivery ratio from sensor nodes to actuator in high mobility, i.e. when the patient is running. There is significant difference between data delivery by multi-radio-multi-channel mechanism and single-radio-multi-channel. The reason is that when human body is in fast motion, there is significant drop in data delivery in single-radio due to single channel assignment to a radio.

Figure 3 represents energy consumption during transmission of data from sensors to actuators. It is observed that single-radio consume less energy as compared to multi-radio multi-channel mechanism. The reason is that, in multi-radio multi-channel, extra radio are participating in WBAN operations.

Figure 4 represents end-to-end delay when human body is

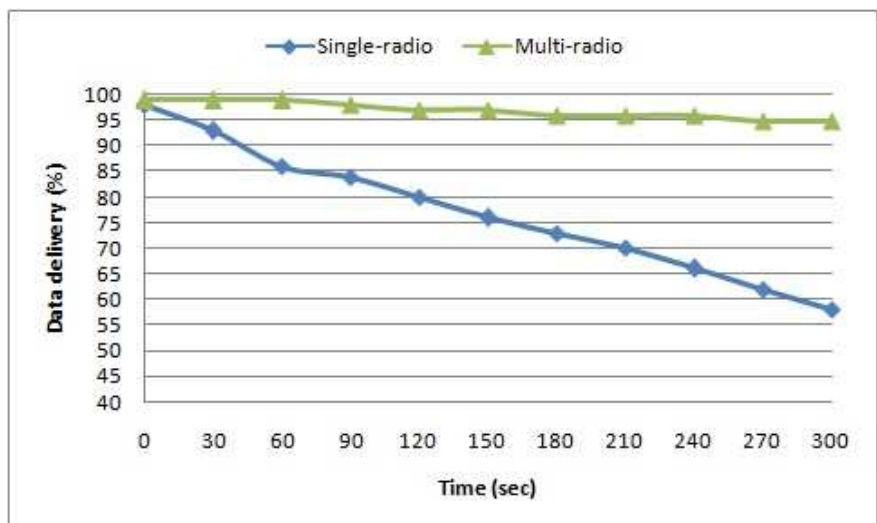

Fig. 2: Data delivery rates comparison of single-radio and multiradio

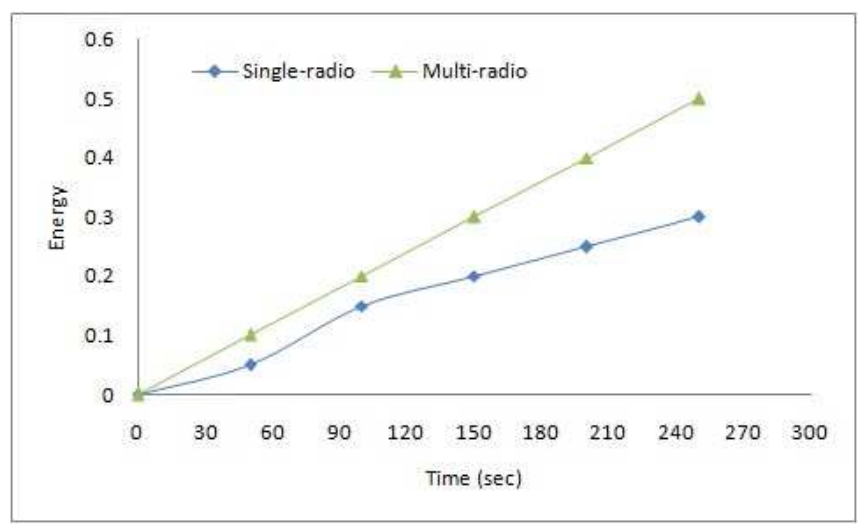

Fig. 3: Energy consumption comparison of single-radio and multi-radio

in motion. As multi-radio multi-channel have many non overlapping channels which significantly reduced end-to-end delay as compared to single-radio.

The results show many advantages such as:

-Multi-radio mechanism has more data delivery rate as compared to single-radios

-Multi-radio mechanism has less end to end delay as compared to single-radios

-Multi-radio mechanism can perform well in case of mobility or interference

-Energy consumption of multi-radio multi-channel mechanism is higher as compared to single radio mechanisms

The results are summarized in the table 1 . 


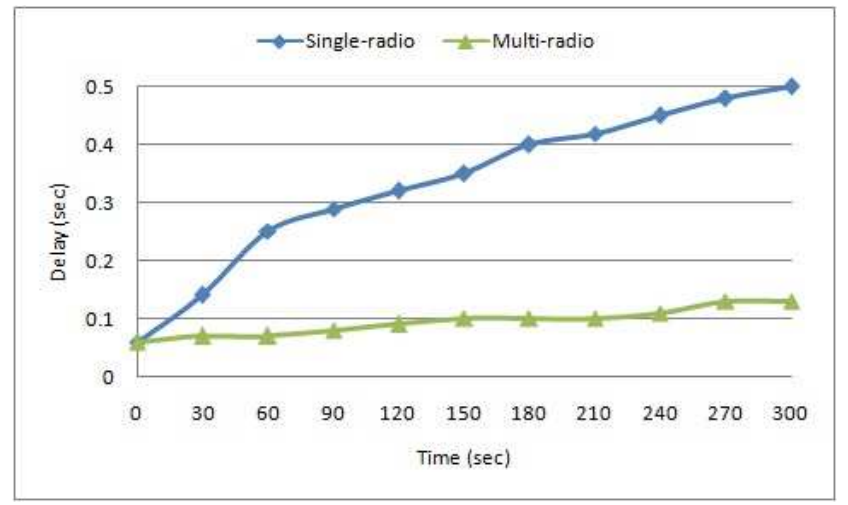

Fig. 4: End-to-end delay of single radio and multi radio mechanisms in case of body motion

Table 1: Comparison of single-radio and multi-radio mechanisms

\begin{tabular}{|l||c|c|}
\hline Features & Single-Radio & Multi-Radio \\
\hline \hline Data-Delivery Rate & Less & More \\
End-to-End Delay & More & Less \\
Energy Consumption & Less & More \\
Interference Effect & More & Less \\
Noise Effect & More & Less \\
Shadowing \& Fading Effect & More & Less \\
\hline
\end{tabular}

\section{Conclusion and future work}

This paper is concentrated on the mechanism of multi-radio multi-channel utilization in WBAN. This paper analyzed the possibility of deploying multi-radio multi-channel scheme in health monitoring. Existing schemes suffers from many constraints such as significant signal loss due to absorption of electromagnetic waves in body fluid, fading, shadowing and interferences. Multi-radio multi-channel can address many of these limitations. Our results show that multi-radio multi-channel offer efficient data delivery rate and reduced end-to-end delay. However, more energy consumption is observed in multi-radio multi-channel mechanism due to operation of extra radios. We are planning to device a mechanism of efficient energy conservation in multi-radio multi-channel mechanism as energy is one of the important factor in resource constraint networks.

\section{Acknowledgement}

The authors extend their appreciation to the Research Centre, College of Applied Medical Sciences and the Deanship of Scientific Research at King Saud University for funding this research.

\section{References}

[1] K. V. Dam, S. Pitchers, and M. Barnard. Body area networks: Towards a wearable future. Proceedings of WWRF kick on meeting, 2, ( 2001).

[2] R. Schmidt, T. Norgall, J. Mcorsdorf, J. Bernhard, and T. Von. Body area network key infrastructure element for patient-centered medical applications. Biomedical engineering, 47, 365-368 (2002).

[3] Body sensor networks. http://ubimon.doc.ic.ac.uk/bsn/m621.html.

[4] Q. Tang, N. Tummala, S. K. S. Gupta, and L. Schwiebert. Communication scheduling to minimize thermal effects of implanted biosensor networks in homogeneous tissue. IEEE Transactions on Biomedical Engineering, 52, 1285-1294 (2005).

[5] A. Bag and M. A. Bassiouni. Energy efficient thermal aware routing algorithms for embedded biomedical sensor networks. IEEE Conference on Mobile Adhoc and sensor systems, 604-609 (2006).

[6] T. Watteyne, S. Blum, M. Dohler, and D. Barthel. Anybody: a self-organization protocol for body area networks. Second International Conference on Body Area Networks, (2007).

[7] B. Latre and et al. A survey on wireless body area network. Journal of wireless networks, 17, (2011).

[8] T. Zasowski, G. Meyer, F. Althaus, and A. Wittneben. Uwb signal propagation at the human head. IEEE Transactions on Microwave Theory and Techniques, 2006, 113-121 (2006).

[9] A. Fort, C. Desset, J. Ryckaert, P. De Doncker, L. Van Biesen, and P. Wambacq. Characterization of the ultra wideband body area propagation channel. IEEE International Conference ICU, (2005).

[10] M. D. Renzo, R. M. Buehrer, and J. Torres. Pulse shape distortion and ranging accuracy in uwbbased body area networks for fullbody motion capture and gait analysis. IEEE Globecom Conference, 3775-3780 (2007).

[11] D. Neirynck. Channel characterisation and physical layer analysis for body and personal area network development. Ph.D. dissertation, (2006).

[12] J. Jung, K. Ha, and J. Lee. Wireless body area network in a ubiquitous healthcare system for physiological signal monitoring and health consulting. International Journal of Signal Processing, Image Processing and Pattern Recognition, 1, 47-54 (2008).

[13] M. Soini, J. Nummela, P. Oksa, L. Ukkonen, and L. Sydanheimo. wireless body area network for hip rehabilitation system. Ubiquitous Computing and Communication Journal, 3, (2008).

[14] B. Latre and et al. A survey on wireless body area network. Journal of wireless networks, 17, (2011).

[15] C. Otto, A. Milenkovic, C. Sanders, and E. Jovanov. system architecture of a wireless body area sensor network for ubiquitous health monitoring. Journal of Mobile Multimedia, 4, 307-326 (2006).

[16] M. A. Hanson and et al. Body area sensor networks challenges and opportunities. IEEE Computer Magazine, 16, 171-193 (2011).

[17] M. Chen and et al. Body area networks a survey. Springer journal of Mobile Netw Appl, 3775-3780 (2007).

[18] S. Khan. Routing security in wireless mesh networks. $P h D$ dissertations, 1-114 (2011). 
[19] A. Raniwala and T. Chiueh. Architecture and algorithms for an ieee 802.11-based multi-channel wireless mesh network. Proceedings of IEEE Conference INFOCOM, (2005).

[20] S. M. Das, H. Pucha, D. Koutsonikolas, Y. C. Hu, and D. Peroulis. Dmesh: Incorporating practical directional antennas in multichannel wireless mesh networks. IEEE J. Select. Areas Commun, 24, 2028 (2006).

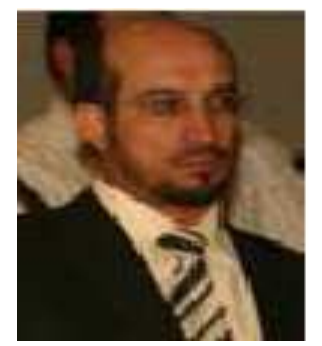

Nabil Ali Alrajeh obtained his Ph.D. in biomedical Informatics engineering from Vanderbilt University, USA. Currently, he is an associate professor at Biomedical Technology Department, King Saud University. He worked as a senior advisor for the Ministry of Higher Education, Saudi Arabia. His research interests include E-health Applications, Hospital Information Systems, Telemedicine, Intelligent Tutoring Systems and Wireless Sensor Network.

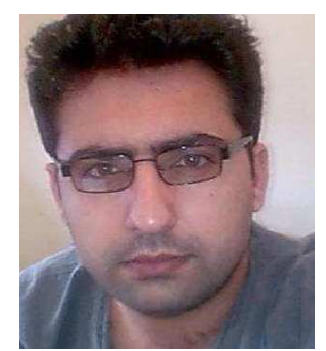

Shafiullah
Khan
obtained his PhD degree
in wireless networks
security from Middlesex
University UK. He is
affiliated with the Institute
of Information Technology,
Kohat University of Science
and Technology, Pakistan
as an Assistant Professor. His research mainly focuses on wireless broadband network architecture, security and privacy, security threats and mitigating techniques. Dr. Khan is Editor in Chief of International Journal of Communication Networks and Information Security.

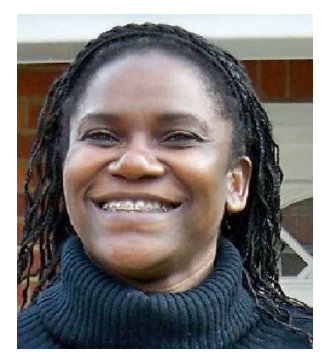

Carlene E-A Campbell has completed her Ph.D from Middlesex University UK in 2011. She is currently serving as Lecturer in Computer Networks at Swansea Metropolitan University, United Kingdom. Her research interests include wireless sensor networks, wireless body area networks, mobile ad hoc networks, networks modeling and simulations.

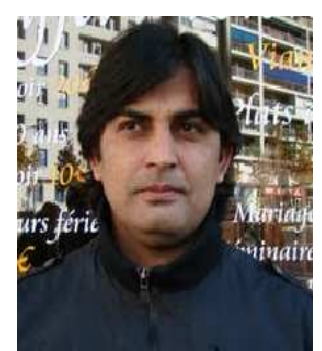

Bilal Shams is Assistant Professor of Information Technology at Kohat University of Science and Technology, Pakistan. He obtained his Ph.D. degree in Information and Coding Theory from University of Cergy-Pontoise, France. His areas of research are digital communication, networks and Error correction codes. 